\title{
When Your Patient's Symptoms do not Fit with Common Neurological Conditions, Consider Rare Diagnoses in the Differential Diagnosis
}

\author{
Patricia Abbott* \\ Department of Nursing, University of Colorado, USA
}

*Corresponding author: Patricia Abbott, Department of Nursing, University of Colorado, USA.

Received Date: November 21, 2019

Published Date: December 04, 2019

\section{Introduction}

An adage all health care providers learn is "When you hear hoofbeats look for horses not zebras." This is an appropriate way to approach patient care but can limit consideration of differential diagnoses. When patients present to a health care provider, the provider gets a history of the present concern and that helps to guide the exam. From this information a differential diagnosis list is determined to determine the most likely diagnosis. In that process of ruling in and ruling out the differentials, if symptoms do not fit, consider if a rare diagnosis could be a possibility.

\section{Case}

63 yo male, 3.5 months post decompression surgery for Cauda Equina Syndrome with a successful recovery, developed sudden onset progressive loss of neurological function and sensation in lower extremities with progressive pain. After two weeks of progressive symptoms, multiple MRIs were done to investigate cause of symptoms. MRI of lumbar spine showed nerve root clumping at L2-4, consistent with arachnoiditis. Surgeon who did decompression surgery stated findings on MRI were normal post-surgery. Neurologist who saw patient felt pain was more likely from muscle spasms and not arachnoiditis. Five weeks after symptoms began, the primary care provider reviewed MRIs, compared to MRIs prior to surgery (no arachnoiditis noted prior to surgery) and did research into arachnoiditis. After diagnosis was made, treatment with Prednisone 50mg qd and Gabapentin was initiated. Progression of pain and neuro function improved but did not resolve. Delay in diagnosis may have resulted in ongoing neurological dysfunction and chronic pain.

Back to those horses and zebras. Progressive loss of neuro function and progressive pain, 3.5 months after a good recovery from lumbar decompression did not fit with "normal findings postsurgery" or "muscle spasms". The "zebra" (arachnoiditis) was in the MRI report when the surgeon and the neurologist saw the patient, but the diagnosis which helped to guide management, was delayed for over a month. Whereas neurological function improved with treatment, patient did not return to baseline and has chronic pain.

When patients do not improve or whose symptoms do not "fit" a common neurological condition, rare dis-eases should be considered to prevent delay in diagnosis, treatment and to minimize decline in neurological function. On the National Institute of Health and the Genetic and Rare Diseases (GARD) websites, a clinician can find a diagnostic support tool (FindZebra) to help them determine if a rare disease may be a diagnostic possibility. Findzebra, (http:// www.findzebra.com/) is a product from Denmark developed by a multidisci-plinary team led by Ole Winther and Mads Emil Matthiesen. (http://www.findzebra.com/).

The purpose of this entry is not intended to define and describe arachnoiditis, the pathophysiology and the presenting symptoms. The intent is to encourage all clinicians to consider a rare disease if the patient does not present with or respond to treatment for common neurological disorders. Early diagnosis and management may limit neurological decline and chronic pain. NIH (2019) https://rarediseases.info.nih.gov/guides/pages/159/findzebradiagnosis-assist-tool

\section{Acknowledgement}

None.

\section{Conflict of Interest}

No conflict of interest. 